\title{
Die ISAR-REACT-2-Studie
}

\section{Intracoronary Stenting and Antithrombotic Regimen-Rapid Early Action for Coronary Treatment}

Hintergrund und Problemstellung: Wie ISAR-REACT-1 gezeigt hat, bringt die zusätzliche Gabe von Abciximab nach Vorbehandlung mit einer Initialdosis von $600 \mathrm{mg}$ Clopidogrel bei Patienten mit stabiler KHK und niedrigem bzw. mittlerem thrombotischen Risiko keinen zusätzlichen Vorteil. Im Vergleich zu Patienten mit stabiler KHK benötigen Patienten mit akutem Koronarsyndrom aufgrund der bei ihnen bestehenden erhöhten Thrombozytenaktivierung eine intensivere Aggregationshemmung. Bislang gab es keine Studie, die

\begin{tabular}{|c|c|c|}
\hline \multicolumn{3}{|l|}{ Methodik } \\
\hline Studiendesign & \multicolumn{2}{|c|}{$\begin{array}{l}\text { Plazebokontrollierte, randomisierte, doppelblin } \\
\text { de, internationale Multicenterstudie }\end{array}$} \\
\hline Primärer Endpunkt & \multicolumn{2}{|c|}{$\begin{array}{l}\text { Kombination aus Tod, Myokardinfarkt, dring- } \\
\text { liche, erneute Revaskularisation des Zielgefäßes } \\
\text { innerhalb von } 30 \text { Tagen nach der PCI }\end{array}$} \\
\hline Patientenauswahl & \multicolumn{2}{|c|}{$\begin{array}{l}\text { Einschlusskriterien: Ruheangina innerhalb der } \\
\text { letzten } 48 \mathrm{~h} \text { mit erhöhtem Troponin } \\
\text { (>0,03 } \mathrm{g} \text { /l) oder ST-Strecken-Senkung } \\
\text { Ausschlusskriterien: akuter ST-Hebungsinfarkt, } \\
\text { hämodynamische Instabilität, Perikarditis, } \\
\text { erhöhtes Blutungsrisiko, maligne Erkrankungen, } \\
\text { relevante hämatologische Erkrankungen, be-- } \\
\text { kannte Allergien auf die Studienmedikation }\end{array}$} \\
\hline $\begin{array}{l}\text { Patientenanzahl in } \\
\text { den randomisierten } \\
\text { Untergruppen }\end{array}$ & $\begin{array}{l}\text { Plazebo } \\
\mathrm{n}=1010\end{array}$ & $\begin{array}{l}\text { Abciximab } \\
\mathrm{n}=1012\end{array}$ \\
\hline $\begin{array}{l}\text { Anteil von Patienten mit } \\
\text { klinischem Follow-up } \\
\text { zum Zeitpunkt des } \\
\text { primären Endpunkts }\end{array}$ & $100 \%$ & $100 \%$ \\
\hline
\end{tabular}

Ergebnisse: $\left({ }^{*} p<0,05\right)$

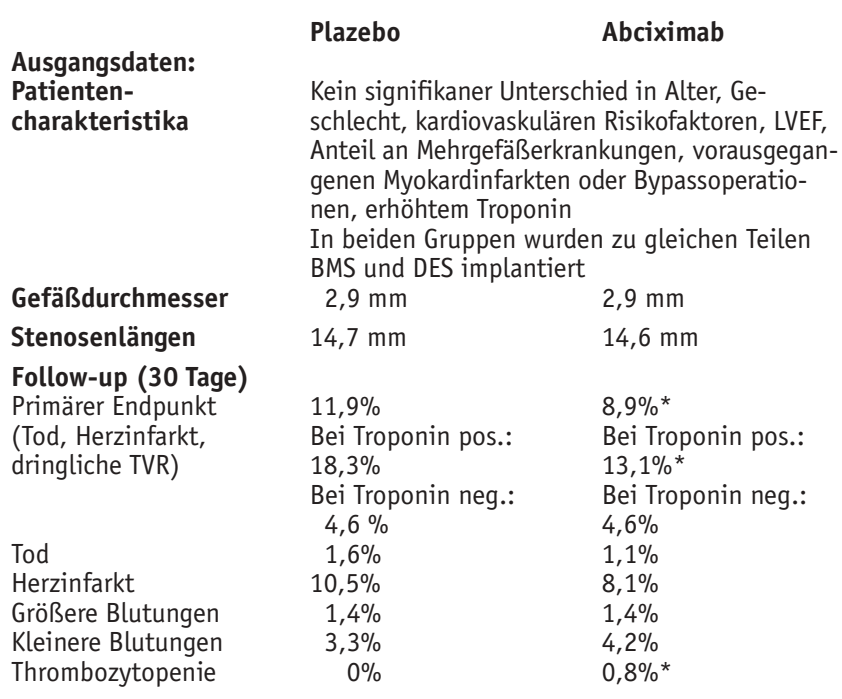

Herz 2006;31:168

DOI $10.1007 / \mathrm{s} 00059-006-2810-1$ bei Patienten mit akutem Koronarsyndrom ohne ST-Hebung nach $600 \mathrm{mg}$ Initialdosis Clopidogrel den zusätzlichen Wert von Abciximab untersuchte. Ziel der Studie war die Prüfung der Hypothese, dass Abciximab bei Patienten mit akutem Koronarsyndrom ohne ST-Strecken-Hebung zusätzlich zu einer 600 mg Initialdosis von Clopidogrel, welche mindestens $2 \mathrm{~h}$ vor der PCI verabreicht wurde, einen klinisch relevanten, protektiven Wert aufweist. Studienleiter waren A. Kastrati und A. Schömig, München.

\begin{tabular}{|c|c|c|}
\hline \multicolumn{3}{|c|}{ Evidence-based-Medicine (EBM-) Score (www.TCTMD.com) } \\
\hline Klinischer primärer Endpunkt & $\mathrm{Ja}=3$, nein $=0$ & 3 \\
\hline Doppelblind (einschließlich Ärzten) & $\mathrm{Ja}=1$, nein $=0$ & 1 \\
\hline $\begin{array}{l}\text { Beobachtungsintervall für den } \\
\text { primären Endpunkt } \geq 6 \text { Monate }\end{array}$ & $\mathrm{Ja}=1$, nein $=0$ & 0 \\
\hline Multicenter (mindestens 3 Zentren) & $\mathrm{Ja}=1$, nein $=0$ & 1 \\
\hline $\begin{array}{l}\text { Externes und vom Steering } \\
\text { Committee unabhängiges Clinical } \\
\text { Event Committee / DSMB (Daten- } \\
\text { sicherheit Monitoring Board) }\end{array}$ & $\mathrm{Ja}=1$, nein $=0$ & 1 \\
\hline Primärer Endpunkt erreicht & $\mathrm{Ja}=1$, nein $=0$ & 1 \\
\hline $\begin{array}{l}\text { Power von } \geq 80 \% \text { für den } \\
\text { primären Endpunkt erreicht }\end{array}$ & $\mathrm{Ja}=1$, nein $=0$ & 1 \\
\hline $\begin{array}{l}\text { Anteil des Follow-up der Patienten } \\
\text { für angiographischen primären } \\
\text { Endpunkt } \geq 80 \% \text { und } \geq 95 \% \text { für } \\
\text { klinischen primären Endpunkt }\end{array}$ & $\mathrm{Ja}=1$, nein $=0$ & 1 \\
\hline \multicolumn{2}{|c|}{$\begin{array}{l}\text { Gesamt-EBM-Score) } \\
\text { („Silber-Score“ für randomisierte Studien) }\end{array}$} & 9 \\
\hline
\end{tabular}

Schlussfolgerung und Kommentar: Bei Patienten mit akutem Koronarsyndrom ohne ST-Strecken-Hebung und hohem thrombotischen Risiko reduziert die zu Clopidogrel (Initialdosis $600 \mathrm{mg}$ ) zusätzliche Gabe von Abciximab das Auftreten relevanter klinischer Ereignisse in einem Zeitraum von $30 \mathrm{Ta}-$ gen nach PCI. Der Vorteil von Abciximab beschränkt sich auf Patienten mit positivem Troponin.

CAPTURE war die erste randomisierte Studie, die einen signifikanten Vorteil von Abciximab bei Patienten mit akutem Koronarsyndrom dokumentierte. Allerdings wurde in CAPTURE das Abciximab 18-24 h vor der PCI gegeben. Die Stentrate war mit ca. 7,6\% für heutige Verhätnisse sehr niedrig, und Clopidogrel wurde damals (1993-1995) nicht verabreicht. ISAR-REACT-2 spiegelt die heutige reale Situation der PCI beim akuten Koronarsyndrom wider und prüfte zum ersten Mal den zusätzlichen Nutzen von Abciximab nach einer Clopidogrel-Vorbehandlung mit einer Initialdosis von 600 mg. Die Stentrate betrug 97\%. Der primäre Endpunkt wurde erreicht. Überraschend fand sich bei den Patienten mit normalem Troponin, die somit „lediglich“ eine ST-Strecken-Senkung aufwiesen, kein Effekt. Bei den Patienten mit erhöhtem Troponin kam es ohne Abciximab in 18,3\% zu schwerwiegenden klinischen Ereignissen. Die NNT (Number Needed to Treat) beträgt für Patienten mit erhöhtem Troponin 18. 